\title{
Unpersönliches Schreiben in der Wissenschaft: Eine Bestandsaufnahme ${ }^{1}$
}

\author{
Mathilde Hennig und Robert Niemann
}

\section{Zusammenfassung}

Der vorliegende erste Teil eines zweiteiligen Aufsatzes beschäftigt sich aus formaler und funktionaler Perspektive mit den sprachlichen Mitteln für einen unpersönlichen Ausdruck beim wissenschaftlichen Schreiben (= Deagentivierungsmittel). Das Formeninventar dieser Deagentivierungsmittel wird zunächst in einem Überblick über bisherige Forschungsbeiträge vorgestellt und anschließend in einem Systematisierungsvorschlag geordnet. Darauf folgen Überlegungen zu einem funktional differenzierten Gebrauch von Deagentivierungsmitteln, wobei der Fokus zum einen auf der Sprecherbezogenheit und zum anderen auf den zugrundeliegenden Handlungstypen liegt. Teil I stellt somit zunächst den Gegenstandsbereich des unpersönlichen Schreibens vor und nimmt dabei noch nicht unmittelbar Bezug auf die Relevanz dieses Phänomenbereichs für Deutsch als Fremdsprache. Eine diesbezügliche Perspektivierung der Thematik erfolgt in einem weiteren Beitrag mit einem interkulturellen Kompetenzvergleich.

\section{Einleitung}

(1) Aus diesem Belegkorpus habe ich aus 10 gleichmäßig über das Korpus verteilten Stellen jeweils 200 Belege exportiert. Dieses 2.000'er Belegkorpus habe ich schließlich bereinigt, indem ich etwa Fälle wie nahm das Kind an die Hand ausgeschlossen habe. Von dem bereinigten Belegkorpus habe ich

${ }^{1}$ Es handelt sich hier um den ersten Teil eines aus zwei Teilen bestehenden Beitrags. Der zweite Teil mit dem Titel »Unpersönliches Schreiben in der Wissenschaft: Kompetenzunterschiede im interkulturellen Vergleich « erscheint in einer späteren Nummer. Wir möchten an dieser Stelle den anonymen Gutachtern für ihre wertvollen Hinweise zu beiden Textteilen danken. 
schließlich 100 gleichmäßig über das Korpus verteilte Belege als Datenmaterial für die Analyse genommen. Wie können die Bereinigungsausschlüsse gerechtfertigt werden? [...] Es wird davon ausgegangen, dass der Phraseologismus als Ganzes eine Bedeutung entfaltet. Bei verbalen Phraseologismen ist diese Bedeutung kategorial als Szene erfassbar. Die Grundfrage lautet: Welche Szene eröffnet der verbale Phraseologismus? Die Antwort findet sich in dem unmittelbaren Kotext des Phraseologismus. (Staffeldt 2011: 198).

Bei diesem Beispiel handelt es sich um ein Standardbeispiel deutscher geisteswissenschaftlicher Wissenschaftssprache. Der Verfasser tritt hier teilweise direkt in Erscheinung - immerhin dreimal verweist er auf die eigene Person mit dem Personalpronomen ich. Er verwendet aber auch Formen, die es ihm ermöglichen, ohne expliziten Verweis auf die eigene Person eigene Handlungen zu schildern: Das Partizipialattribut bereinigt, die Passivformen wird ausgegangen und können gerechtfertigt werden sowie ist erfassbar und die Reflexivkonstruktion findet sich. Interessant ist an diesem Beispiel gerade der Wechsel zwischen agenshafter und nicht agenshafter Verfasserreferenz:

»Gemeinsam ist beiden Optionen, daß der Bezug zum Verfasser, funktional gesehen, signalisiert wird; sie unterscheiden sich darin, daß bei der Selbstbenennung der Bezug strukturell erfolgt (d.h. der Handlungsträger wird mittels einer sprachlichen Struktur markiert), während bei der fehlenden Selbstbenennung der Handlungsträger strukturell eliminiert wird.« (Kresta 1995: 92).

Trotz der beschriebenen funktionalen Äquivalenz scheint keine völlige Wahlfreiheit zu herrschen, vielmehr besteht im Sinne von Diewald (2008) »kommunikative Obligatorik « (vgl. Fußnote 1 auf S. 446): »In den modernen Fachsprachen hat die Vermeidung der ersten Person Singular als eine zentrale stilistische Verpflichtung zu gelten, mit welcher der Objektivitätsanspruch und die allgemeine Gültigkeit der fachlichen Äußerungen hervorgehoben werden.« (Roelcke 2010: 83). Diese kommunikative Obligatorik, die Schreiber von wissenschaftlichen Texten offenbar dazu veranlasst, sich häufiger für nicht agenshafte als für agenshafte Verfasserreferenz zu entscheiden, kann mit den konventionalisierten Anforderungen an wissenschaftliche Texte erklärt werden. So ist es nicht nur in der Wissenschaftskommunikationsforschung ein Gemeinplatz, dass Wissenschaft und folglich auch Wissenschaftssprache sachbezogen, objektiv und intersubjektiv zu sein habe. ${ }^{1}$ Das Beispiel illustriert aber gerade, dass das offenbar nicht mehr automatisch zu einer vollständigen Vermeidung von ich führen muss, wie es Weinrich 1989 provokativ als »Ich-Verbot« beschrieben hat:

1 Vgl. zur (u. a. terminologischen und begrifflichen) Geschichte der wissenschaftlichen Objektivität die wissenschaftsgeschichtlichen Arbeiten von Daston (u. a. 2002). 
»Erstes Verbot: Ein Wissenschaftler sagt nicht >ich`. Auszählungen [...] haben ergeben, daß die Ich-Form eine außerordentlich niedrige Frequenz hat [...] Das kann man wohl nicht anders als mit einem Ich-Verbot erklären, das allerdings so gut wie nirgendwo explizit ausgesprochen ist, sondern stillschweigend aus dem Gebot der wissenschaftlichen Objektivität abgeleitet wird. Denn die Tätigkeit des Wissenschaftlers soll ja von den individuellen Besonderheiten der einzelnen Forscherpersönlichkeit unabhängig sein, sie muß ferner jederzeit intersubjektiv nachgeprüft werden können [...].« (Weinrich 1989: 132f.).

Aus dem Gebot der Objektivität und Intersubjektivität lässt sich das Postulat der Anonymität ableiten: "Anonymität hat, besonders in der Wissenschaftssprache, die Funktion, die an einen Autor gebundene Subjektivität zu eliminieren und den Wahrheitsgrad sowie die Objektivität und mögliche Allgemeingültigkeit der fachbezogenen Aussagen zu verstärken.«(Oksaar 1998: 397). Für den Autor hat das weitreichende Konsequenzen:

»Thus, starting from the assumption that academic discourse should be as objective as possible, the author is expected to remain hidden behind the facts, research results, tables, figures, formula and the like, to keep his/her personality as unobtrusive and incospicuous as possible and practically >disappear< from the text.« (Vassileva 2000: 9).

Theo Bungarten spricht von "sprachlicher Entfremdung in der Wissenschaft« (1989):

»Das Prinzip der Rationalität und Objektivität ist das oberste Gebot. Bereits in der Platonischen Ideenlehre kann man den Ursprung des Strebens nach rationaler und objektiver Erkenntnis sehen: in der Idee spielt das menschliche Subjekt des Erkenntnisprozesses keine Rolle. Nur die ratio mit ihren denkerischen Fähigkeiten ist gefragt. Alle anderen menschlichen Eigenschaften, Gebundenheiten und Befindlichkeiten werden vernachlässigt. Die ratio wird in das Bild des objektiven Wissenschaftlers eingelagert, ja mit ihm und einigen instrumentellen Fähigkeiten, die für die wiss. Arbeit notwendig sind, gleichgesetzt. Der solcherart von allen menschlichen `Unzulänglichkeiten befreite objektive Wissenschaftler kommuniziert mit seinem ebenso objektiven Widerpart ausschließlich auf sachlicher, rational-argumentativer, logischer Ebene.« (Bungarten 1989: 23).

Bungarten schlussfolgert: »In dieser Welt der Wissenschaft, in dieser Welt von aufgehenden und fallenden Sternen am Theorienhimmel, von Erkenntnisblitzen, von Milchstraßen und Schwarzen Löchern, droht der Wissenschaftler als Mensch zum rationalen Wesen zu verkrüppeln« (1989: 35).

Dem Bild des objektiven Wissenschaftlers steht aber die autorschaftliche Verantwortung gegenüber: »Jede wissenschaftliche Wissensproduktion unterliegt [...] ausdrücklich der autorschaftlichen Verantwortung." (Steiner 2009: 11). Steiner leitet daraus das »Ich-Problem« ab: »Wie kann man in einem Text den Platz des forschenden und erkennenden Wissenschaftlers einnehmen und gleichzeitig den Ansprüchen an eine subjektunabhängige Aussagekonsistenz gerecht werden?« 
(2009: 15). Schließlich setzt jede wissenschaftliche Autorentätigkeit »eine autorschaftliche Passion voraus« (Steiner 2009: 11):

»Für die wissenschaftlichen Disziplinen ist Erkenntnis immer vorläufige Erkenntnis. Für eine einzelne Autorin, einen einzelnen Autor besteht aber die Aufgabe gerade darin, sich mit einer Textproduktion auf Aussagen festzulegen, den autorschaftlichen Prozess der Erkenntnisgenerierung als abgeschlossen und beigebrachte Begründungen als zureichend zu apostrophieren. [...] Etwas wissen zu wollen, heisst mit Blick auf die Verbreitung von Erkenntnis, das Wagnis einzugehen es als Wissen zu behaupten (Kant).« (Steiner 2009: 11).

Das Bild von einem objektiven Wissenschaftler, der von seiner institutionalisierten Beobachterposition her die Welt betrachtet, wissenschaftliche Erkenntnisse daraus rein sach- und gegenstandsbezogen ableitet und durch das Verfassen wissenschaftlicher Texte der Menschheit zur Verfügung stellt, ist also ganz offensichtlich eine Vereinfachung, die verschleiert, dass es im Wissenschaftsbetrieb ja gerade um die Rolle einzelner Wissenschaftler im wissenschaftlichen Diskurs geht. Mindestens ebenso wichtig wie die Gegenstandsorientierung ist die Gruppenorientierung, wie von Polenz treffend feststellt: "Man glaubt über Sachen und zum Zwecke von Arbeit oder Information zu reden und weiß nicht (oder würde es womöglich nicht zugeben), dass man damit auch Gruppenstatus und Gruppendynamik signalisiert.« (von Polenz 1981: 87). So handelt es sich bei wissenschaftssprachlicher Tätigkeit »stets um spezielle Tätigkeiten von Gruppen, bei denen die Demonstration von Gruppenzugehörigkeit nach innen und außen eine Rolle spielt« (von Polenz 1981: 87).

Gruppenzugehörigkeit (Hoffmann 1989 spricht von einem »soziolinguistischen Differential«) ist gekoppelt an einen bestimmten gruppenspezifischen Habitus, welcher akzeptable Handlungsweisen ermöglicht (vgl. Teil II). Dieser Habitus und somit Gruppenzugehörigkeit müssen allerdings erst erworben werden. Dass Wissenschaftssprache desto mehr "zum Gemeingut der ganzen Gesellschaft" wird, »je stärker [...] Wissenschaft und Technik das Leben aller Menschen durchdringen, je höher in diesem Zusammenhang das Bildungsniveau steigt« (Hoffmann 1989: 80), ${ }^{1}$ führt noch nicht automatisch zu einer Erweiterung der Gruppe der Wissenschaftler. Um nicht nur rezeptiv, sondern auch produktiv am Wissenschaftsgeschehen teilzuhaben, bedarf es mindestens der folgenden Kennzeichen der »sozialen Gemeinschaft« bzw. »scientific community«: »eine fachwissenschaftliche Ausbildung, wissenschaftlich-theoretische Interessen, besondere

1 »Natürlich folgt die Gesellschaft nicht in ihrer Gesamtheit der Logik der Wissenschaft, aber kaum jemand wird ernsthaft bezweifeln wollen, dass wissenschaftliches Wissen eine größere Rolle in modernen Gesellschaften spielt als je zuvor in der Geschichte. Die Wissensgesellschaft ist somit wohl besser als eine > verwissenschaftlichte $<$ denn als eine Wissenschaftsgesellschaft charakterisiert.«(Weingart 2003: 8). 
Institutionen, gemeinsamer Bestand an Methoden und Theorien, wissenschaftliche Hilfsmittel, Normen und Verhaltensweisen, und die jeweilige Wissenschaftssprache (Bungarten 1989: 31).

In der universitären Ausbildung werden Studenten an das Verfassen von wissenschaftlichen Texten herangeführt. Es geht dabei um den Erwerb eines wissenschaftssprachlichen Habitus (vgl. Teil II). Sie stehen dabei vor der gewaltigen Aufgabe, die noch nicht vollständig ausgeprägte Gruppenzugehörigkeit durch die Verwendung solcher sprachlichen Mittel zu kompensieren, die geeignet erscheinen, den transitorischen Stand des Erwerbsprozesses zu verschleiern und einen an einer Zielnorm orientierten Kompetenzstand zu suggerieren. Es liegt auf der Hand, dass für nicht-muttersprachliche Studenten auch noch die Notwendigkeit von sprachlichem und kulturellem Kompetenzerwerb hinzukommt, sodass das wissenschaftliche Schreiben für sie eine noch größere Anforderung darstellt (vgl. Teil II).

Zwar spricht von Polenz von einer »leichte[n] Nachahmbarkeit von wissenschaftssprachlichen Stilmitteln« (1981: 94), es stellt sich jedoch die Frage, ob den skizzierten Anforderungen tatsächlich allein durch eine Imitation wissenschaftssprachlicher Kompetenz durch eine Verwendung von einschlägig erscheinenden Ausdrucksformen an der Oberfläche begegnet werden kann (vgl. Teil II). Gerade in einem stark sozial und kulturell determinierten Kommunikationsbereich wie dem der Wissenschaftskommunikation ist die Funktionalität der verwendeten Ausdrucksmittel nicht zu unterschätzen. Mit anderen Worten: Wenn zwar durchaus damit gerechnet werden kann, dass sich Studenten im Erwerbsprozess die einschlägigen Ausdrucksmittel zu eigen machen, kann dadurch allein noch nicht der Abstand zwischen Novizen und Experten nivelliert werden (vgl. Teil II).

Der vorliegende Beitrag wurde aus Platzgründen in zwei Teile aufgeteilt. Der vorliegende erste Teil bietet eine Übersicht über sprachliche Realisierungsformen des unpersönlichen Schreibens (= Deagentivierungsmuster) und die einschlägigen funktionalen Zusammenhänge. Teil I stellt somit zunächst den Gegenstandsbereich des unpersönlichen Schreibens vor und nimmt dabei noch nicht unmittelbar Bezug auf die Relevanz dieses Phänomenbereichs für Deutsch als Fremdsprache.

Im zweiten Teil des vorliegenden Beitrags betrachten wir Kompetenzunterschiede im unpersönlichen Schreiben im interkulturellen Vergleich. Wir werden dort die allgemeine Frage nach Kompetenzunterschieden zwischen Experten und Novizen einerseits und muttersprachlichen und nicht-muttersprachlichen Novizen andererseits mit der spezifischen Frage nach sprachlichen Realisierungsformen des unpersönlichen Schreibens verbinden, weil wir davon ausgehen, dass das Verhältnis von expliziter und impliziter Verfasserreferenz, d. h. der Umgang mit der Diskrepanz zwischen dem Anonymitätspostulat und der verantwortungsvollen Autorschaft, ein Paradegebiet für die Untersuchung von Erwerbsphänomenen im Bereich wissenschaftlicher Schreibkompetenz ist. 


\section{Deagentivierung formal und funktional}

\subsection{Formeninventar}

Ausgehend davon, dass sprachliche Mittel zum Ausdruck von Objektivität, Anonymität (bzw. Origo-Exklusivität, vgl. Czicza/Hennig 2011: 52) »auf der lexikalischen, morphosyntaktischen und textuellen Ebene analysiert werden « können (Oksaar 1998: 397), möchten wir zunächst betonen, dass es im Folgenden ausschließlich um morphosyntaktische Mittel geht.

Autoren wissenschaftlicher Texte sind handelnde Personen in mehrfacher Hinsicht: »Die >Einsehbarkeit< von Forschungshandlungen ergibt sich erst dadurch, dass wissenschaftliche Autoren Forschungs- und Erklärungshandlungen in einem Textkommunikat verschränken.« (Steiner 2009: 10). Wie nun kann ein Autor seine Forschungs- und Erklärungshandlungen transparent machen, wenn er doch eigentlich als Autor an der Textoberfläche möglichst wenig in Erscheinung treten sollte? Die Schlüsselstelle für die Bearbeitung dieser Frage sind offenbar Handlungsprädikate, die »systemhaft primär an die satzsemantische Bedingung geknüpft [sind], den Tiefenkasus (Kasusrolle) AGENTIV (»Agens« in der traditionellen Grammatik) zu bezeichnen, der mit intentional handelndes Wesen (Mensch oder Tier)< definiert wird « (von Polenz 1981: 97). von Polenz bezeichnet die Vermeidung der expliziten Nennung eines Agens als Deagentivierung:

"Die Bezeichnung des AGENTIVs von Handlungsprädikaten kann nun in der deutschen wie wohl in den meisten Sprachen in verschiedenen Arten und Stufen weggelassen bzw. verschoben werden. Die Gesamtheit dieser Möglichkeiten heiße De- oder Entagentivierung von Handlungsprädikaten.« (von Polenz 1981: 97).

von Polenz benennt als einschlägige grammatische Muster der Deagentivierung das Passiv, man-Sätze (»in denen der AGENTIV nur noch formal ausgedrückt und auf eine Spezifizierung nach Personen oder Gruppen verzichtet oder diese ausgeschlossen wird « (1981: 98)), Nominalisierungen sowie den »Subjektschub«, den er als »die sublimste Art und semantisch folgenreichste Stufe von Entagentivierung « beschreibt: ${ }^{1}$

»Hier haben wir es vielmehr mit der stilistischen Erscheinung zu tun, daß statt einer systematisch primären, eigentlichen Ausdrucksweise eine systematisch sekundäre, uneigentliche gewählt wird. Anstelle der primären (von der satzsemantischen Klasse

${ }^{1}$ Bereits dem Titel des Aufsatzes von von Polenz »Über die Jargonisierung von Wissenschaftssprache und wider die Deagentivierung « ist ein deutlich sprachkritischer Tenor zu entnehmen: von Polenz verharrt in seinem Aufsatz nicht in der Rolle des deskriptiv orientierten Beobachters, sondern er warnt ausdrücklich vor den Konsequenzen der Jargonisierung: »[...] wo also der Jargon nicht mehr durchschaut wird, besteht die Gefahr, daß die Konsumenten des Bildungsjargons das selbständige >Handeln`von Abstrakta entnehmen und das implizite Handeln von Menschen aus dem Blick verlieren« (1981: 107). 
des Prädikats geregelten) Bezeichnung der ersten Prädikationsstelle werden zum Zwecke sprachökonomischer Verkürzung die Bezeichnungen anderer Prädikationsstellen in die Subjektstelle des Satzes ‘geschoben «.« (von Polenz 1981: 100).

(2) Das Projekt verfolgt [Subjektschub] zwei Ziele: ein theoretisches und ein semantisch-lexikographisches. (Staffeldt 2011: 193).

Besonders häufig ist dieser Subjektschub in Abstracts anzutreffen, in denen gerne Formulierungen wie »Der folgende Beitrag analysiert ...« verwendet werden. Dorgeloh/Wanner sprechen deshalb in ihrer empirisch-diachronen Studie zur englischen Wissenschaftssprache von einer "paper construction« (2009: 530).

Deagentivierung hat sich in der noch vergleichsweise jungen Geschichte der Wissenschaftskommunikationsforschung bereits als beliebter Forschungsgegenstand erwiesen. Das liegt sicherlich einerseits an der klaren Beziehbarkeit auf pragmatische Bedingungen der Wissenschaftskommunikation wie die Gebote der Objektivität, Intersubjektivität und Anonymität (Bungarten 1989: 34; Oksaar 1998; Kaiser 2002: 138), andererseits aber auch an der guten Zugänglichkeit im Rahmen einer oberflächenorientierten Textanalyse. Als einschlägige Deagentivierungsmuster wurden bislang vorrangig untersucht (ohne Anspruch auf Vollständigkeit):

- Beneš (1966): Passiv, man-Sätze, nominal-verbale Konstruktionen, possessive Passivformen (»sie erhalten das bezahlt«);

- Schwanzer (1981): Passiv, man, Infinitivkonstruktionen mit ist/sind/hat/hat $m a n+z u+$ Infinitiv (hier: Halbmodale), Funktionsverbgefüge, von Verben abgeleitete Substantivierungen (hier: deverbale Nominalisierungen), gerundivische Attribute (= Halbmodale als Partizipialattribute), lassen + sich + Infinitiv;

- Kresta (1995): Passiv, Ablativ-Subjekte (= Subjektschub) ${ }_{1}^{1}$ sei(en) + Partizip, infinite Formen;

- Oksaar (1998): Passiv, Passiv mit Modalverben, sich lassen + Infinitiv, sein + zu + Infinitiv (hier: Halbmodale), sein + Adjektiv mit den Suffixen -bar,-sam,-lich, Verbalabstrakta, Funktionsverbgefüge, verbfreie Kurzsätze;

- Kaiser (2002): Passiv- und Passivperiphrasen, Reflexivkonstruktionen, unpersönliche Konstruktionen, man, Infinitivkonstruktionen, deverbalisierte Adjektive, Ellipsen (etwa: hier ein Beispiel zu);

- Steinhoff (2007): Meinungsausdrücke, Passiv + Modalverb, lassen- und Reflexivkonstruktionen, Subjektschub;

- Schloemer (2012): Meinungsausdrücke, man, Passiv- und Passivperiphrasen, Subjektschub, PI-Konstruktionen, spezifische Valenzrealisierungen (etwa: es muss nicht verwundern).

1 Zu den Motiven für die Umbenennung siehe Kresta (1985: 86 f.). 
Der hier vorzustellende Ansatz unternimmt den Versuch, verschiedene Deagentivierungsmuster zu systematisieren. Den Ausgangspunkt bildet die Überlegung, dass Deagentivierungsbemühungen erhebliche Konsequenzen für die gesamte grammatische Organisation von wissenschaftlichen Texten haben: Ein Satz mit einer finiten Verbform enthält automatisch die grammatische Kategorie Person. Es handelt sich dabei im Sinne von Diewald um eine sprachinterne Obligatorik: »wenn Form X, dann Form Y« (Diewald 2008: 9). ${ }^{1}$ Wenn ein Verweis auf das Agens einer Äußerung vermieden werden soll, müssen deshalb Strategien zum Tragen kommen, die es ermöglichen, das »intentional handelnde Wesen«, das hinter der durch ein Handlungsprädikat ausgedrückten Handlung steht, und die am Verb markierte Kategorie Person nicht aufeinander zu beziehen. Einerseits sind diverse Strategien zu beobachten, das Agens auszublenden (Passiv) oder zu verlagern (Subjektschub), also trotz der Beibehaltung der verbalen Kategorie Person die Nennung des Handlungsträgers zu umgehen:

(2) Das Projekt verfolgt [Subjektschub] zwei Ziele: ein theoretisches und ein semantisch-lexikographisches. (Staffeldt 2011: 193).

(3) Es werden also 2,5 Millionen für die Ausrüstung der Polizei ausgegeben [Passiv], um diese fit zu machen. (Staffeldt 2011: 199).

Andererseits bietet sich der Rückgriff auf Strukturen ohne finites Verb an: Wenn kein finites Verb vorhanden ist, entfällt der Zwang, ein mit diesem Verb kongruierendes Subjekt zu benennen:

(3) Es werden also 2,5 Millionen für die Ausrüstung der Polizei ausgegeben [Passiv], um diese fit zu machen [Infinitivkonstruktion]. (Staffeldt 2011: 199).

(4) Im letzten Absatz taucht die FDP zunächst nur tief eingebettet [Partizipialkonstruktion] (FDP-Wahlkämpfe, FDP-Rechenschaftsberichte) oder nur angedeutet [Partizipialkonstruktion] (eine reiche Partei; das Parteiengesetz) auf. (Vinckel-Roisin 2011: 388).

${ }^{1}$ Diewald geht es gerade darum, diesem in der Grammatikalisierungsforschung zentralen Typ der Obligatorik das Prinzip der kommunikativen Obligatorik gegenüberzustellen: »Wenn Intention X, dann Form Y « (Diewald 2008: 10). Das unpersönliche Schreiben in der Wissenschaft ist ein Musterbeispiel für die kommunikative Obligatorik: Wenn du Anforderungen an wissenschaftliches Schreiben wie Anonymität und Objektivität gerecht werden willst, dann wähle Formen des unpersönlichen Schreibens. Das steht nicht im Widerspruch zum oben hergestellten Zusammenhang zwischen Finitheit und der grammatischen Kategorie Person: Wenn man sich für ein Deagentivierungsmuster entscheidet, folgt man zunächst dem Prinzip der kommunikativen Obligatorik. Die genaue Ausgestaltung der deagentivierenden Sprachmittel unterliegt dann aber wie jegliche sprachliche Äußerung Prinzipien der sprachinternen Obligatorik. 
Neben der Unterscheidung zwischen »Vermeidung und Beibehaltung der Kategorie Person« bietet sich als weiterer Klassifikationsaspekt die Unterscheidung zwischen verbaler und nominaler Organisation der Deagentivierung an: Es ist allgemein bekannt, dass in der Fach- und Wissenschaftssprache eine »Informationsverlagerung vom Verb zum Nomen « (Kretzenbacher 1991: 122) beobachtet werden kann. ${ }^{1}$ Gerade in Bezug auf die Deagentivierung liegt es auf der Hand, nominale Organisationsformen (deverbale Nominalisierung, Partizipialattribut) zu wählen, da auch auf diese Weise die Notwendigkeit einer Subjektrealisierung entfällt:

(5) Olsen zufolge wird die vom Basisverb geforderte [Partizipialattribut] Richtungsergänzung durch die Partikel auf erbracht [Passiv], und zwar ohne explizite Nennung [deverbale Nominalisierung] des internen Arguments der P-Relation. (Bryant 2011: 60)

Wenn man nun die Unterscheidung zwischen Beibehaltung und Vermeidung der verbalen Kategorie Person sowie die Unterscheidung zwischen nominaler und verbaler Organisation korreliert und einzelne Deagentivierungsmuster den dadurch gewonnenen Klassen zuordnet, so ergibt sich folgendes Bild:

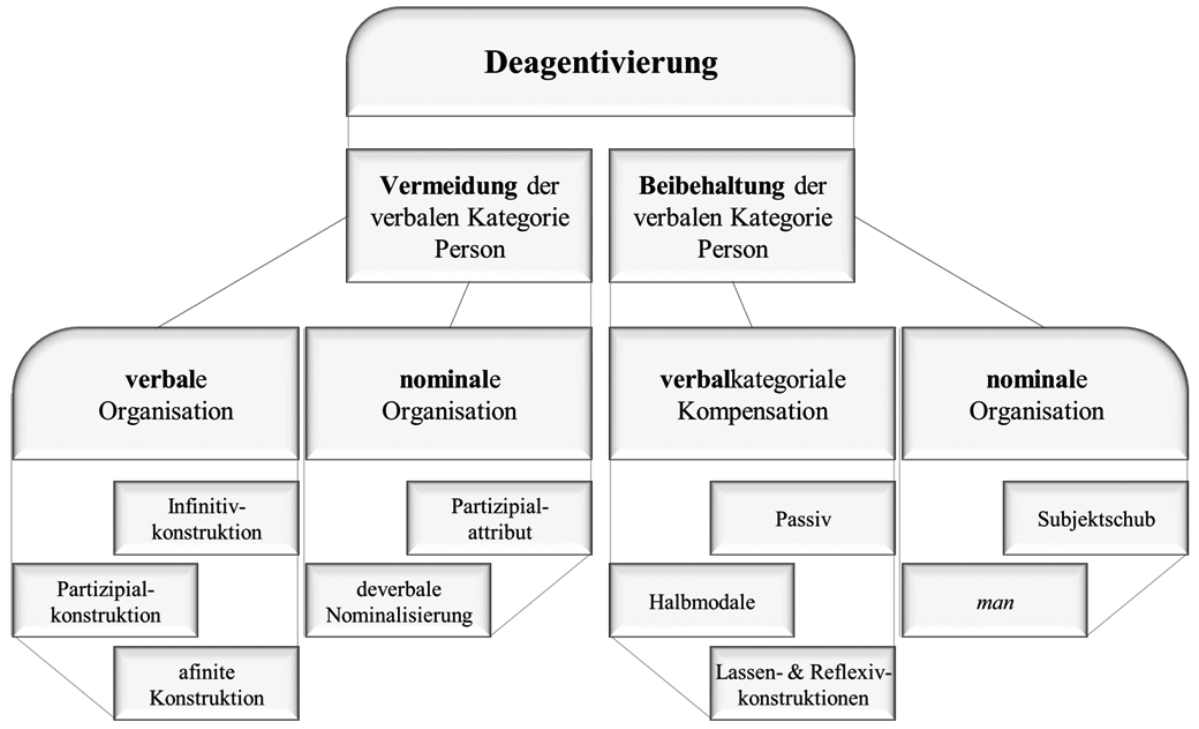

Übersicht 1: Deagentivierungsmuster

${ }^{1}$ Vgl. auch bereits Beneš: »Die reichliche Auffüllung der Satzglieder und starke Belastung des einfachen Satzes haben zwangsläufig zur Folge, daß das Verb schon rein quantitativ zurücktritt.« (1966: 28). 
Die vorgestellte Systematik zu Deagentivierungsmustern erhebt keinen Anspruch auf Vollständigkeit, sondern soll vielmehr prinzipielle Möglichkeiten einer systematischen Kategorisierung aufzeigen. Das folgende Beispiel soll die zentrale Rolle der Deagentivierung für das wissenschaftliche Schreiben noch einmal abschlieBend illustrieren:

(6) Eine der zentralen Fragestellungen der hier präsentierten [Partizipialattribut] Studie lautet daher: Wie gelingt dem Zweitspracherwerbenden der Einstieg ins deutsche Lokalisierungssystem und lassen sich qualitative und quantitative Unterschiede in Abhängigkeit der Muttersprache beobachten [Lassen- $\mathcal{E}$ Reflexivkonstruktion]? Zur Beantwortung [deverbale Nominalisierung] des zweiten Teils der Frage wurden zwei typologisch sehr verschiedene L1-Sprachen ausgewählt [Passiv]: [...]. (Bryant 2011: 71)

\subsection{Funktionale Gesichtspunkte}

\subsubsection{Deagentivierung und Origobezug}

In der Einleitung haben wir bereits angedeutet, dass die Verwendung von Deagentivierungsformen allein noch keine wissenschaftliche Schreibkompetenz ausmacht. Wir gehen vielmehr davon aus, dass erst die in einschlägige funktionale Zusammenhänge der Wissenschaftskommunikation eingebettete Verwendung von Deagentivierungsformen kennzeichnend für ein kompetentes Agieren in der Wissenschaft ist. Wir möchten deshalb nun unsere Überlegungen zu einschlägigen funktionalen Zusammenhängen vorstellen.

Den Ausgangspunkt bildet die Einschätzung, dass einzelne Belege für die oben als Deagentivierungsmuster klassifizierten sprachlichen Phänomene eine unterschiedliche Qualität in Bezug auf die Anforderungen der Wissenschaftskommunikation aufweisen können. ${ }^{1}$ Die folgende Übersicht stellt Beispiele aus der Wissenschafts- und Alltagskommunikation gegenüber und fasst die im Anschluss zu erläuternden funktionalen Überlegungen zusammen:

${ }^{1}$ Vgl. Kresta: »Es ist zweifelhaft, ob Verfasser Passivsätze im allgemeinen bewusst ausschließlich dazu einsetzen, um einen Sachverhalt zu entpersönlichen. (1995: 82) Diejenigen Passivverwendungen, bei denen der Verfasser selbst als Urheber der beschriebenen Handlung angesehen werden kann, nennt Kresta »Autoren-Passiv« (1995: 84). 


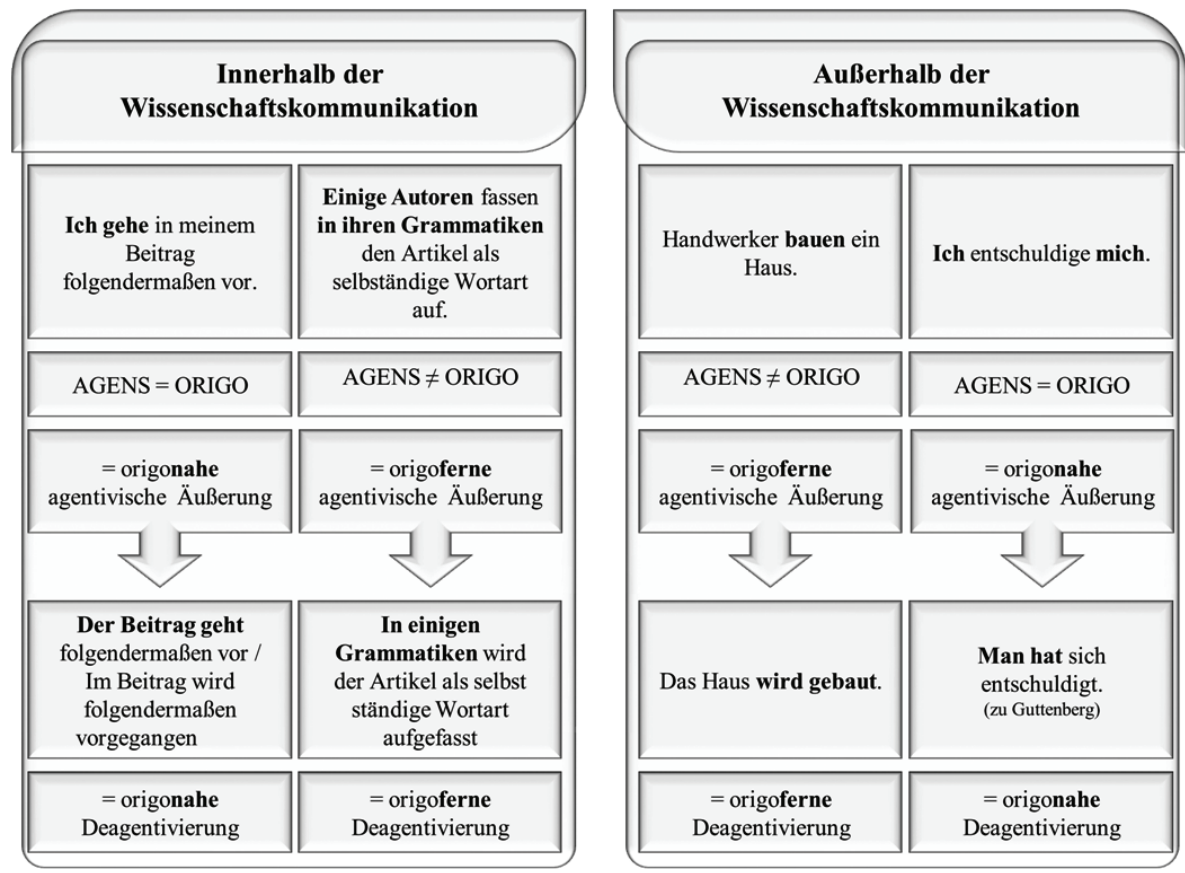

Übersicht 2: Deagentivierung und Origobezug

Die Übersicht ist folgendermaßen zu verstehen: Deagentivierungen haben immer ein agentivisches Pendant. Da im agentivischen Pendant das Agens sichtbar ist, gehen wir hier zunächst von agentivischen Beispielen aus. Wir können nun unterscheiden zwischen solchen agentivischen Äußerungen, in denen Agens und Sprecher übereinstimmen (Agens = Origo), und solchen, in denen das Agens eine nicht mit dem Sprecher übereinstimmende Person ist (Agens $\neq$ Origo $)^{1}$. Mit Handlungsprädikaten können folglich origonahe und origoferne Äußerungen verbunden sein und zwar sowohl innerhalb als auch außerhalb der Wissenschaftskommunikation. Wenn man nun die obigen Beispiele deagentiviert, ergibt sich auch eine Unterscheidung zwischen origonaher und origoferner Deagentivierung. In gewisser Weise fin-

${ }^{1}$ Mit Origo rekurrieren wir auf den Ausgangspunkt des Zeigfeldes der Sprache, also auf die bekannte hier-jetzt-ich-Origo aus Karl Bühlers Zweifelderlehre (1934/1999: 102-120 und 1936: 11). Bühler sagt in diesem Zusammenhang Folgendes: »Das Feld des Zeigens, ein Koordinatensystem mit der Origo hier, jetzt, ich bringt jeder mit; es ist unveräußerlich für jeden wachen Menschen, der >bei sich « ist« (1936: 11). Wir verwenden den Terminus Origobezug für Sprecherbezug, weil wir den auf den Autor hinweisenden Charakter bestimmter Deagentivierungsausdrücke akzentuieren möchten. 
det sich hierin Weingartens (1994) Unterscheidung zwischen Objektivitätsstil und Handlungsstil in der Wissenschaftssprache wieder. Wir haben demzufolge einen Handlungsstil, wenn der Untersuchungsgegenstand als Hervorbringung von Sprechern dargestellt ist, und einen Objektivitätsstil, wenn Inhalte unabhängig von Sprechern dargestellt werden (vgl. 1994: 125 und 133). Der hier implizierte Zusammenhang von Handlung und Origobezug wird von uns in Abschnitt 2.2.2 noch in einem weiteren Zusammenhang thematisiert werden.

Wir meinen, dass es ein entscheidender Unterschied ist, ob in wissenschaftlichen Texten durch eine Deagentivierung die Person des Autors überlagert wird oder ob einfach ein anderer Urheber einer Handlung nicht genannt wird, weil er möglicherweise nicht zentral für die Argumentation ist. Wir halten es auch für wahrscheinlich, dass gerade die origoferne Deagentivierung die von von Polenz angesprochene »leichte Nachahmbarkeit von wissenschaftssprachlichen Stilmitteln« (1981: 94) ermöglicht.

Origoferne Deagentivierungen können mit der von Polenz'schen Unterscheidung zwischen gegenstands- und gruppenorientierter fachsprachlicher Kommunikation (1981: 86) als rein gegenstandsorientiert bewertet werden: Es handelt sich hierbei nicht um sprachliche Mittel, die eingesetzt werden, um dem in der Einleitung beschriebenen Dilemma zwischen dem Postulat der Anonymität und verantwortungsvoller Autorschaft gerecht zu werden. Mit anderen Worten: Der Autor spielt bei diesen Deagentivierungen keine Rolle. Origonahe Deagentivierungen werden hingegen eingesetzt, um Autorhandlungen zu beschreiben und trotzdem auf die Nennung des Autors zu verzichten (vgl. die Beispiele in der Übersicht). Der Autor orientiert sich hier also an den Konventionen der Gruppe. Gruppenzugehörigkeit kann sich auch darin manifestieren, dass mit einer Deagentivierung nicht nur der Autor als Bezugspunkt der Handlung überlagert wird, sondern dass die Origo quasi durch die Gruppe erweitert wird. Wir verwenden zur Erfassung dieses Phänomens im Folgenden das Konzept der >community :
»The concept of community draws attention to the idea that we do not use language to communicate with the world at large, but with other members of our social groups, each with its own norms, categorizations, sets of conventions and ways of doing things. [...] in engaging with others we enter into a community of shared belief or value concerning what is interesting or worth discussing [...] The notion of community therefore seeks to offer a framework within which these actions occur and so characterize how speakers position themselves with and understand others. [...] Community conventions are therefore also a means of fostering group mytholo- gies, solidarity and social control, helping to ringe-fence communities by identifying their users as insiders and excluding others.« (Hyland 2009: $47 \mathrm{f}$.).

Deagentivische Bezüge auf die Origo des Sprechers als Bestandteil der scientific community bezeichnen wir im Folgenden als >origo + community $<$. Das folgende Beispiel soll dies illustrieren: 
(7) Die optionale PP kann sich, wie in (20) und (21) geschehen, dann der reduzierten P-Relation als Modifikator anbieten. Natürlich stellt sich die Frage, warum das interne Argument erst unterdrückt und dann mit einer pleonastischen PP wieder expliziert wird. Olsen gibt hierfür eine typologische Erklärung: [...]. (Bryant 2011: 60).

In diesem Beispiel geht das `Sich fragen über den Autor hinaus: Zum einen wird mit dem konzessiven „Natürlich“ ein gemeinsamer und scheinbar selbstverständlicher Wissenshintergrund in der community suggeriert, vor dem sich die Frage nicht nur für den Autor selbst stellt; zum anderen wird durch den Folgesatz (»Forscher X erklärt dies folgendermaßen«) deutlich, dass sich bereits andere Forscher mit der Frage beschäftigt haben und sich die Frage folglich auch anderen stellt und gestellt hat.

\subsubsection{Deagentivierung und sprachliche Handlung}

»Die Bestimmung der illokutiven Qualität von Texten erweist sich spätestens auf den zweiten Blick als keine leichte Aufgabe.«(Ehlich 1993: 24). Zu einer sach- und gegenstandsorientierten Wissenschaftssprache passt der Illokutionstyp Assertion:

»Eine zentrale Funktion wissenschaftlicher Texte besteht in der Weitergabe von Wissen bzw. in dessen Expansion zu neuem Wissen. [...] Diejenigen sprachlichen Handlungen, die auf das engste mit der Weltwiedergabe-Funktion des Wissens zu tun haben, sind offensichtlich die Assertion und die Frage." (Ehlich 1993: 24). ${ }^{1}$

Ehlich weist ausgehend von Beispieldiskussionen die Einordnung wissenschaftssprachlicher Handlungen als assertiv als »gängiges Vorverständnis« zurück: "[...] so stoßen wir faktisch auf jene zweite Struktur, die es nicht mit der reinen Abbildung von Wirklichkeit in Text, sondern mit der Abbildung einer diskursiven Struktur in eine textuelle Struktur zu tun hat « (1993: 28). Diese zweite Struktur bezeichnet Ehlich als »eine Struktur des Streitens, als eine eristische Struktur [...], denn sie enthält Bewertungen von vorgängigen Auffassungen, Qualifizierungen als >echt< bzw. > unecht« « (1993: 28 f.). Die eristische Struktur bietet »eine Relativierung der ganzen wissenschaftlichen Erkenntnisgewinnung an einen fortlaufenden Prozeß, der in sich im Streit der wissenschaftlichen Auffassungen entwickelt wird « (1993: 29).

${ }^{1}$ Da bei Ehlich die Frage in der weiteren Argumentation keine wesentliche Rolle mehr spielt und für die Erfassung zentraler Handlungsformen bei Ehlich nur die dichotomische Gegenüberstellung von auf die Weltwiedergabe-Funktion bezogenen Handlungen (er spricht von einer »Familie der assertiven illokutiven Typen«, 1993: 29) und nicht auf diese Funktion beziehbaren Handlungen zentral ist, verzichten auch wir im Folgenden auf eine weitere Berücksichtigung dieses Illokutionstyps. 
Wir gehen nun davon aus, dass gruppenorientierte, origonahe Deagentivierungen als Bestandteil dieser eristischen Struktur interpretiert werden können: Auch hier geht es nicht um reine Assertion, sondern um die Abbildung »diskursiver Kennzeichen der wissenschaftlichen Praxis in die Textstruktur (Ehlich 1993: 28). Die Grundannahme ist, dass ein origonaher Ausdruck eben durch den Origobezug nicht rein assertiv, d. h. weltwiedergebend bzw. Wirklichkeit abbildend sein kann. Die (vermeintliche) Unmittelbarkeit bei der Weltwiedergabe wird durch den Origobezug des Autors gebrochen und somit zu einer vermittelten Wiedergabe. Die Vermittlung durch den Autor führt dazu, dass die Äußerung s seinsgebunden und somit schon immer aus einer bestimmten Perspektive bzw. von einem bestimmten Standpunkt aus getätigt wird; folglich ist so eine Äußerung in gewisser Weise immer diskursiv. ${ }^{1}$ Vor diesem Hintergrund nehmen wir bei origonahen Deagentivierungen (mit Ausnahme von textorganisierenden Handlungen s. u.) einen eristischen und bei origofernen einen assertiven Gehalt an. Die folgenden Beispiele sollen eine gegenstandsorientierte, assertive Deagentivierung und eine gruppenorientierte, eristische Deagentivierung gegenüberstellen:

(8) Durch die Aktivierung des sprechersalientesten Referenten an der rechten Satzperipherie wird dem Leser klar gemacht, um wen es sich hier handelt. (Vinckel-Roisin 2011: 391).

(9) Für Hand kann hier Aktion oder Tätigkeit o.ä. als Zieldomäne einer eventuellen konzeptuellen Metonymie angenommen werden. (Staffeldt 2011: 212).

Während wir in Beispiel 9 mit annehmen eine origonahe, diskursive Handlung haben, die durch Vorgangspassiv mit Modalverb deagentiviert wird, werden in Beispiel 8 mit der deverbalen Nominalisierung Aktivierung und dem Vorgangspassiv wird ... klar gemacht Aussagen mit Weltwiedergabe-Funktion getroffen. Hier haben wir folglich keinen Origobezug.

Für unseren Zusammenhang möchten wir Ehlichs Auffassung, dass man mit der assertiven und eristischen Struktur auf »eine Art illokutiver Grunddichotomie stößt, die wissenschaftliche Texte als Texte kennzeichnet« (1993: 26), allerdings noch weiter differenzieren. Wir gehen noch von einem weiteren Handlungstyp aus, der mit den folgenden Beispielen illustriert wird:

1 Der Gedanke der »)Seinsgebundenheit « eines jeden lebendigen Denkens « (Mannheim 1929/1995: 71) ist sozusagen ein Grundgedanke der Wissenssoziologie. Fasst man Wissenschaftler als soziale und handelnde Wesen in sozialen Gemeinschaften (vgl. Teil II), muss man sich stets auch über »den Zusammenhang zwischen sozialer Seinslage und Sicht « (Mannheim 1929/1995: 71) bewusst sein (siehe auch die Anmerkungen zum Denkzwang in Denkkollektiven in Teil II). 
(10) In diesem Kapitel geht es primär darum zu beleuchten, welchen syntaktischen Status das lokale drauf in Äußerungen wie (4) und (5) beansprucht. Hierfür sollen Gemeinsamkeiten und Unterschiede zu den direktionalen Konstruktionen in (20) und (21) [...] herausgearbeitet werden. (Bryant 2011: 59).

(11) Die Daten, die hier besprochen werden, stammen aus einer größer angelegten Studie (Bryant 2010), die den wechselseitigen Spracheneinfluss auf die Entwicklung lokaler Konzepte untersucht, d.h. ein Großteil der Kinder wurde sowohl in der Muttersprache als auch in der Zweitsprache getestet. (Bryant 2011: 70).

Auch bei diesem Handlungstyp haben wir einen Origobezug, sodass es sich nicht um eine reine Weltwiedergabe handeln kann. Allerdings sind diese Handlungen nicht der eristischen Struktur zuzuordnen, da sie primär den eigenen Text bzw. die Projektumgebung eines Autors zum Gegenstand haben: Der Autor bietet hier also Informationen zur Organisation des eigenen Textes (Beispiel 10) sowie Hinweise auf übergeordnete, projektbezogene Handlungen (Beispiel 11). In (10) und (11) liegt also zwar eine origonahe Deagentivierung vor, diese hat aber nicht die gleiche Qualität in Bezug auf die diskursive Funktion von Wissenschaftssprache. Wir werden diese Handlungsform im Folgenden als Textorganisation bezeichnen. Die folgende Übersicht fasst die Handlungsdomänen der Deagentivierung zusammen:

\section{Orientierung}

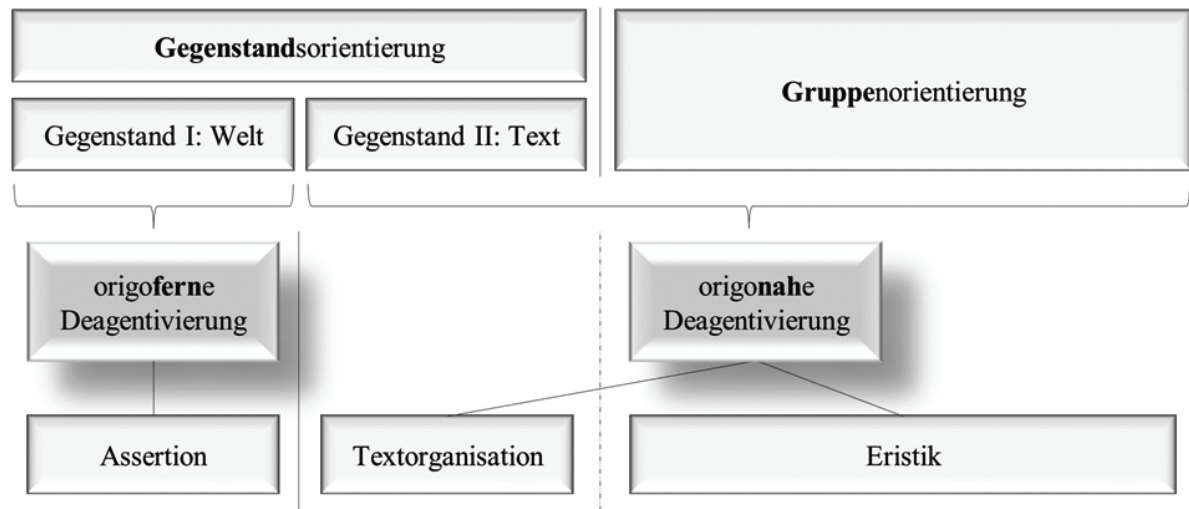

Übersicht 3: Deagentivierung und sprachliche Handlung 


\section{Literatur}

\section{Primärliteratur}

Bryant, Doreen: »Präpositionaladverbien im Erst- und Zeitspracherwerb - Pleonasmen oder Funktionsträger?«, Zeitschrift für Germanistische Linguistik 39 (2011), 55-89.

Staffeldt, Sven: »Die phraseologische Konstruktionsfamilie [X Präp Hand Verb]«, Zeitschrift für Germanistische Linguistik 39 (2011), 188-216.

Vinckel-Roisin, Hélène: »Wortstellungsvariation und Salienz von Diskursreferenten: Die Besetzung des Nachfeldes in deutschen Presstexten als kohärenzstiftendes Mittel«, Zeitschrift für Germanistische Linguistik 39 (2011), 377-404.

\section{Sekundärliteratur}

Beneš, Eduard: »Syntaktische Besonderheiten der deutschen wissenschaftlichen Fachsprache, Deutsch als Fremdsprache 3 (1966), 26-36.

Beneš, Eduard: »Die formale Struktur der wissenschaftlichen Fachsprachen in syntaktischer Hinsicht«. In: Bungarten, Theo (Hrsg.): Wissenschaftssprache: Beiträge zur Methodologie, theoretischen Fundierung und Deskription. München: Fink, 1981, 185-211.

Bühler, Karl: Sprachtheorie: Die Darstellungsfunktion der Sprache. Mit einem Geleitwort von Friedrich Kainz. 3. Aufl. Stuttgart: Lucius \& Lucius, 1934/1999 (UTB 1159).

Bühler, Karl: »Das Strukturmodell der Sprache«, Travaux du Cercle Linguistique de Prague 6 (1936) (Reprint von 1968), 3-12.

Bungarten, Theo: »Sprachliche Entfremdung « in der Wissenschaft«. In: ders. (Hrsg.): Wissenschaftssprache und Gesellschaft: Aspekte der wissenschaftlichen Kommunikation und des Wissenstransfers in der heutigen Zeit. 2. Aufl. Tostedt: Attikon, 1989, 22-43.

Czicza, Dániel; Hennig, Mathilde: »Zur Pragmatik und Grammatik der Wissenschaftskommunikation«, Fachsprache 33 (2011), 36-60.

Daston, Lorraine: »Eine Geschichte der wissenschaftlichen Objektivität«. In: Mayntz, Renate (Hrsg.): Akteure - Mechanismen - Modelle: Zur Theoriefähigkeit makro-sozialer Analysen. Frankfurt a. M.: Campus, 2002, 44-60.

Diewald, Gabriele: „Grammatikalisierung, Grammatik und grammatische Kategorien: Überlegungen zur Entwicklung eines grammatikalisierungsaffinen Grammatikbegriffs«. In: Stolz, Thomas (Hrsg.): Grammatikalisierung und grammatische Kategorien. Brockmeyer: Bochum, 2008, 1-32 (Diversitas Linguarum, 21).

Dorgeloh, Heidrun: Wanner, Anja: »Formulaic Argumentation in Scientific Discourse «. In: Corrigan, Roberta et al. (Hrsg.): Formulaic Language: Aquisition, Loss, Psychological Reality and Functional Explanations. Vol. 2. Amsterdam: John Benjamins, 2009, 523-544.

Ehlich, Konrad: »Deutsch als fremde Wissenschaftssprache«, Jahrbuch Deutsch als Fremdsprache 19 (1993), 13-42.

Hoffmann, Lothar: »Wissenschaftssprache als gesellschaftliches Phänomen «. In: Bungarten, Theo (Hrsg.): Wissenschaftssprache und Gesellschaft. Aspekte der wissenschaftlichen Kommunikation und des Wissenstransfers in der heutigen Zeit. 2. Aufl. Tostedt: Attikon, 1989, 76-93.

Hyland, Ken: Academic Discourse. English in a Global Context. Chennai: Newgen, 2009.

Kaiser, Dorothee: Wege zum wissenschaftlichen Schreiben: Eine kontrastive Untersuchung zu studentischen Texten aus Venezuela und Deutschland. Tübingen: Stauffenburg, 2002.

Kresta, Ronald: Realisierungsformen der Interpersonalität in vier linguistischen Fachtextsorten des Englischen und Deutschen. Frankfurt a. M.: Lang, 1995 (Theorie und Vermittlung der Sprache, 24). 
Kretzenbacher, Heinz L.: »Syntax des wissenschaftlichen Fachtextes«, Fachsprache 2 (1991), 118-137.

Mannheim, Karl: Ideologie und Utopie. 8. Aufl. Frankfurt a. M.: Klostermann, 1929/1995.

Oksaar, Els: »Das Postulat der Anonymität für den Fachsprachengebrauch«. In: Kalverkämper, Hartwig; Wiegand, Herbert Ernst (Hrsg.): Fachsprachen: Ein internationales Handbuch zur Fachsprachenforschung. Berlin: de Gruyter, 1998, 397-401 (Handbücher zur Sprachund Kommunikationswissenschaft, HSK, 14.1).

von Polenz, Peter: »Über die Jargonisierung von Wissenschaftssprache und wider die Deagentivierung «. In: Bungarten, Theo (Hrsg.): Wissenschaftssprache. Beiträge zur Methodologie, theoretischen Fundierung und Deskription. München: Fink, 1981, 85-110.

Roelcke, Thorsten: Fachsprachen. 3., neu bearb. Aufl. Berlin: Schmidt, 2010 (Grundlagen der Germanistik, 37).

Schloemer, Anne: »Interkulturelle Aspekte der Wissenschaftskommunikation am Beispiel der Textsorte wissenschaftlicher Aufsatz«, Professional Communication and Translation Studies 5 (2012), 48-64.

Schwanzer, Viliam: »Syntaktisch-stilistische Universalia in den wissenschaftlichen Fachsprachen«. In: Bungarten, Theo (Hrsg.): Wissenschaftssprache: Beiträge zur Methodologie, theoretischen Fundierung und Deskription. München: Fink, 1981, 213-230.

Steiner, Felix: Dargestellte Autorschaft: Autorkonzept und Autorsubjekt in wissenschaftlichen Texten. Tübingen: Niemeyer, 2009 (Reihe Germanistische Linguistik, 282).

Steinhoff, Torsten: Wissenschaftliche Textkompetenz: Sprachgebrauch und Schreibentwicklung in wissenschaftlichen Texten von Studenten und Experten. Tübingen: Niemeyer, 2007.

Vassileva, Irena: Who is the Author? A Contrastive Analysis of Authorial Presence in English, German, French, Russian and Bulgarian Academic Discourse. Sankt Augustin: Asgard, 2000 (Sprachen und Sprachenlernen, 309).

Weingart, Peter: Wissenschaftssoziologie. Bielefeld: transcript, 2003.

Weingarten, Rüdiger: »Zur Stilistik der Wissenschaftssprache: Objektivitäts- und Handlungsstil«. In: Brünner, Gisela; Graefen, Gabriele (Hrsg.): Texte und Diskurse: Methoden und Forschungsergebnisse der Funktionalen Pragmatik. Opladen: Westdeutscher Verlag, 1994, 115-135.

Weinrich, Harald: »Formen der Wissenschaftssprache«, Jahrbuch der Akademie der Wissenschaften zu Berlin, 1989, 119-158.

\section{Prof. Dr. Mathilde Hennig}

Professur für Germanistische Linguistik mit dem Schwerpunkt Sprachtheorie und Sprachbeschreibung an der Justus-Liebig-Universität Gießen. Forschungsschwerpunkte: Grammatik des Gegenwartsdeutschen unter Einbeziehung pragmatischer Fragestellungen, Grammatik des Neuhochdeutschen, Nähe-Distanz-Theorie, Wissenschaftskommunikation.

\section{Robert Niemann}

Lehrkraft für besondere Aufgaben an der Justus-Liebig-Universität Gießen mit den Forschungsschwerpunkten Wissenschaftskommunikation und Wissenschaftssprachgeschichte. 



\section{Machen Sie Ihre Lernenden fit für den Beruf!}

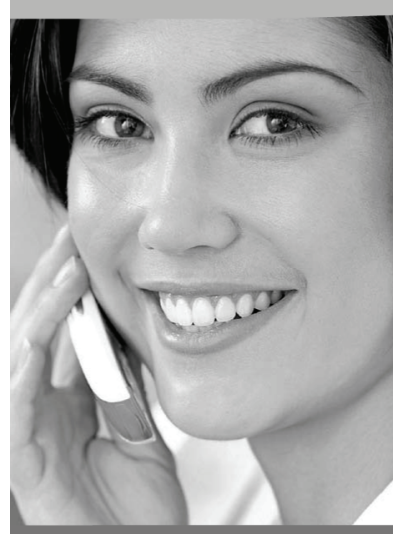

Jetzt durchstarten ...

... im Berufsalltag:

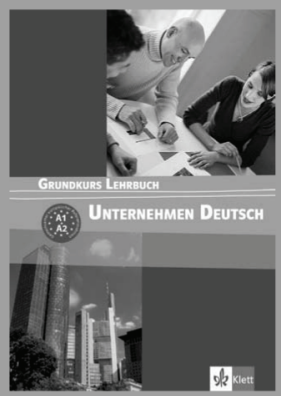

Unternehmen Deutsch

A1-A2 978-3-12-675740-9

B1-B2 978-3-12-675745-4

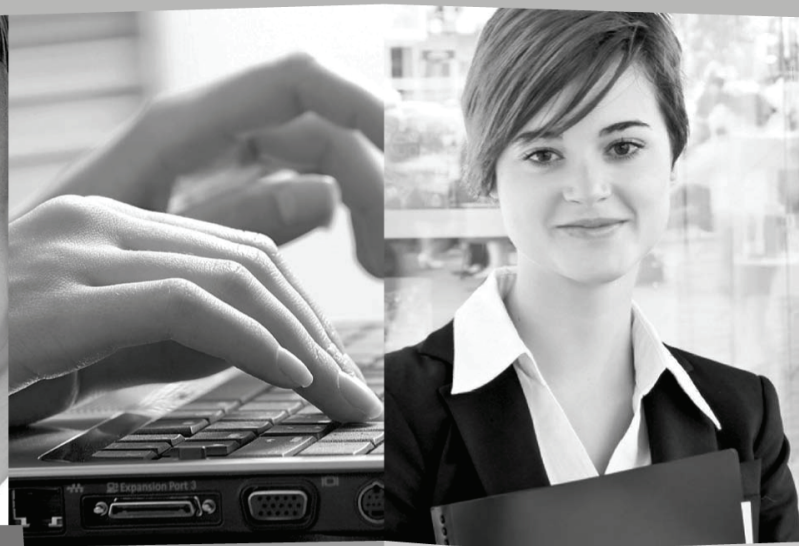

... mit einer erfolgreichen Bewerbung:

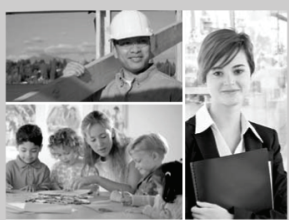

Bewerbungstraining

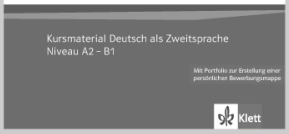

Bewerbungstraining

978-3-12-675243-5
Berufsleben

B1 978-3-12-675725-6
Mehr Informationen unter www.klett.de
Sprachen fürs Leben!

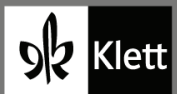




\title{
SILVIA DEMMIG / SARA HÄGI / HANNES SCHWEIGER (HRSG.)
} DACH-LANDESKUNDE

\author{
Theorie - Geschichte - Praxis
}

2013, 252 Seiten, kt.,

29 , - EUR

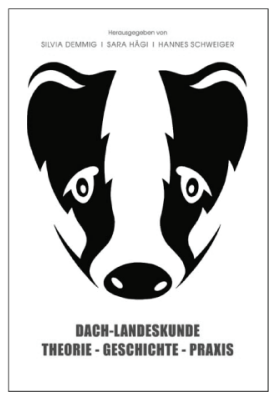

ISBN: 978-3-86205-304-9

Dieser Band bietet die erste ausführliche Bestandsaufnahme zur Theorie, Geschichte und Praxis einer am DACH-Konzept orientierten Landeskunde. Gerade in den letzten Jahren ist viel Bewegung in die Diskussion zur Landeskundedidaktik gekommen und es wird der Vermittlung der Vielfalt und Differenziertheit des deutschsprachigen Raumes im Deutsch als Fremdsprache-Unterricht wieder verstärkt Aufmerksamkeit geschenkt. Die Landeskundedidaktik wurde zudem an die kulturwissenschaftliche Theoriebildung angeschlossen und hat dadurch wichtige neue Impulse erhalten.

Das DACH-Konzept wird in diesem Buch aus unterschiedlichen Perspektiven beleuchtet: aus kulturwissenschaftlicher, linguistischer, sprachenpolitischer und institutioneller. Zudem wird das DACH-Konzept anhand eines Fallbeispiels vor dem Hintergrund der spezifischen Situation des Deutschunterrichts in einem Land (hier: Polen) analysiert. Die Umsetzung in Lehrplänen, Lehrwerken und Unterrichtsmaterialien wird ebenso skizziert wie in Fortbildungsseminaren und in der Ausbildung von DeutschlehrerInnen. Abschließend kommen Fachverbände und Mittlerorganisationen zu Wort und stellen ihren Zugang zur $\mathrm{DACH}$-Landeskunde sowie ihren Beitrag für die Etablierung des DACH-Konzepts in der Praxis dar.

Damit versammelt der Band die Beiträge wichtiger Akteure und wissenschaftlicher ImpulsgeberInnen zur Theorie und Praxis landeskundlichen Lehrens und Lernens auf der Basis des DACH-Konzepts.

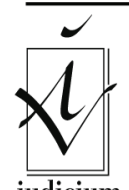

iudicium

\section{IUDICIUM Verlag GmbH}

Dauthendeystr. $2 \cdot \mathrm{D}-81377$ München

Tel.+49(0)89718747·Fax +49(0)897142039·info@iudicium.de

Bestellungen richten Sie bitte an Ihre Buchhandlung oder an den Verlag. 


\section{Machen Sie Ihre Lernenden fit für den Beruf!}

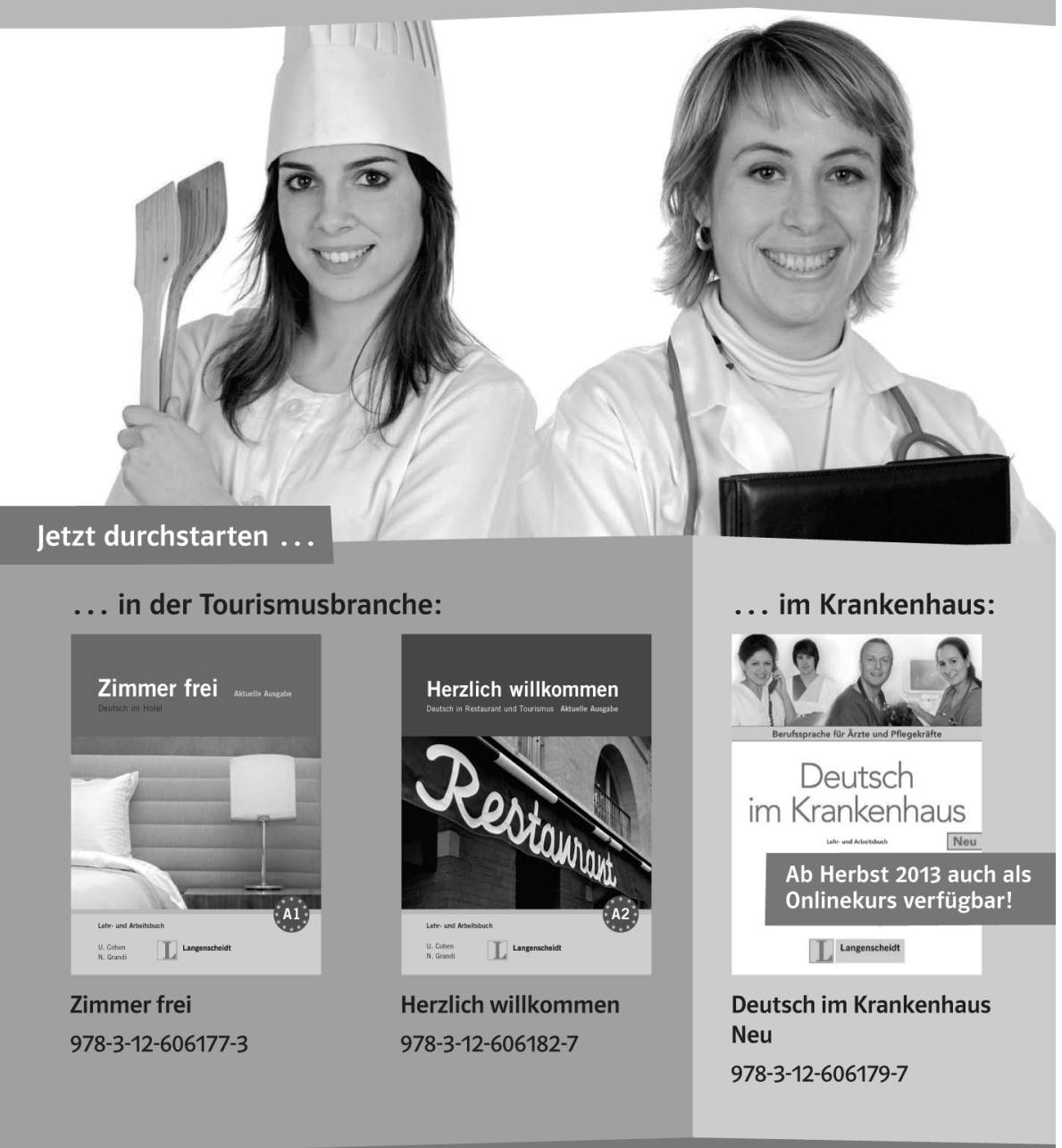

Mehr Informationen unter:

www.klett-langenscheidt.de und www.klett.de 


\section{JAHRBUCH DEUTSCH ALS FREMDSPRACHE INTERCULTURAL GERMAN STUDIES}

herausgegeben von Andrea Bogner, Konrad Ehlich, Ludwig M. Eichinger, Andreas F. Kelletat, Hans-Jürgen Krumm, Willy Michel, Ewald Reuter, Alois Wierlacher, Barbara Dengel (Dokumentation)

Band $38 \cdot 2012$, 306 Seiten, geb. $45,-$ EUR

ISBN: 978-3-86205-352-0

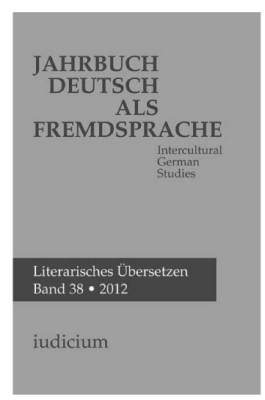

Dass sich die deutsche Literatur durch Übersetzungen aus den Sprachen der Welt immer wieder bereichert hat und weiterhin bereichert, ist ein Gemeinplatz. Dennoch haben diese "importierten" und originär interkulturellen Texte keinen ihrer eminenten Bedeutung entsprechenden Eingang in unsere Forschung und Lehre gefunden. In einem stark interdisziplinär ausgerichteten Themenschwerpunkt werden linguistische, komparatistische, translationswissenschaftliche, fremdsprachenphilologische und übersetzungspoetologische Fragestellungen behandelt. Literaturübersetzer berichten über ihre Erfahrungen, und der Blick fällt auch auf "kleinere" bzw. in der Regel seltener berücksichtigte Sprachen wie das Arabische oder Koreanische.

Mit seinem Themenschwerpunkt lädt das Jahrbuch dazu ein, die ins Deutsche übersetzte Literatur als Gegenstand interkultureller Literaturbetrachtung stärker in den Fokus zu rücken.

\section{Allgemeiner Teil}

Adelheid HU: „On regarde une langue à travers l'autre“. Mehrsprachigkeit als Wert und Herausforderung · Susanne GÜNTHNER: „Doing Culture“ - Kulturspezifische Selbst- und Fremdpositionierungen im Gespräch · Rūta EIDUKEVIČIENÉ: „Die Geschichte kennt keine Gnade.“ Zur Geschichtsproblematik in Josef Haslingers Roman Das Vaterspiel

Thematischer Teil: Literarisches Übersetzen. Herausgegeben von Andreas F. Kelletat

Andreas F. KELLETAT: Wem gehört das übersetzte Gedicht? Zugleich eine Einführung in den Thematischen Teil · Dieter LAMPING: Relationen und Funktionen der literarischen Übersetzung. Eine theoretische Skizze · Henrik NIKULA: Aspekte des literarischen Übersetzens - linguistisch gesehen - Gauti KRISTMANNSSON: Wie man ohne Original übersetzt. Die Rolle der Translation in der Kulturgeschichte · Manfred Peter HEIN: Übersetzen · Michèle MÉTAIL: Übersetzen als Prozess · Mahmoud HASSANEIN: Das Sams spricht Arabisch. Ein Kommentar zur Übersetzung von Paul Maars Eine Woche voller Samstage · Klaus-Jürgen LIEDTKE: Gemeinsame Ostsee - übersetzt und digital. Zu Entstehung und Konzeption einer Virtuellen Ostseebibliothek · Ton NAAIJKENS: Der Literaturübersetzer. Sein Profil im Spannungsfeld von Literatur- und Übersetzungsgeschichte. Die Orchestrierung des Joseph Roth als Beispiel · KIM Nam Hui: Jade Musik/Freude Friede. André Eckardt und die Vermittlung (mündlich tradierter) koreanischer Literatur - Renata MAKARSKA: Wie viel Fremdheit (v)erträgt ein Text? Zur Übersetzung von Mehrsprachigkeit · Gesine Lenore SCHIEWER: Let's Talk About ,Translation'. Translationswissenschaftliche Fragen im Zusammenhang von Text und Diskurs am Beispiel urbaner Innovation

Forum • Jahresbibliographie • Dokumentation • Rezensionen

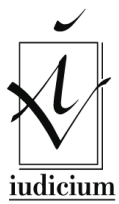

IUDICIUM Verlag GmbH

Dauthendeystr. $2 \cdot \mathrm{D}-81377$ München

Tel. +49 (0)89718747·Fax +49(0)897142039·info@iudicium.de

Bestellungen richten Sie bitte an Ihre Buchhandlung oder an den Verlag.

iudicium

Das Gesamtverzeichnis finden Sie im Internet unter www.iudicium.de 